\title{
CORRESPONDENCE
}

\section{Possible ocular involvement in pulmonary alveolar proteinosis}

\section{To the Editors:}

Pulmonary alveolar proteinosis (PAP) is a rare lung disease characterised by lipoproteinaceous material accumulation in the alveoli. In the most frequent form of the disease (acquired PAP), a neutralising auto-antibody against granulocytemacrophage colony-stimulating factor (GM-CSF) causes defects in the functioning of alveolar macrophages, including impairment of the catabolism of surfactant lipids and proteins [1].

The clinical manifestations of PAP are progressive dyspnoea, cough or fatigue. Available reports have never disclosed any ocular involvement.

Herein, we describe, in a patient with acquired PAP, a retinal lesion resembling adult-onset foveomacular vitelliform dystrophy (AOFVD), a macular degeneration characterised by a generally autosomal dominant inheritance pattern and yellowgrey macular deposits in the deeper retinal layers.

A 47-year-old male was referred to the Dept of Ophthalmology (University of Ferrara, Ferrara, Italy) due to a progressive loss of vision in his right eye. His medical history was positive for the recent onset of acquired PAP, which was treated with a therapeutic whole-lung lavage. At the ophthalmological examination, his best-corrected visual acuity was 20/32 in the right eye and 20/20 in the left eye. Ophthalmoscopic evaluation was unremarkable in the left eye, whereas the right eye revealed a round-shaped, yellowish subfoveal lesion. On fluorescein angiography, this lesion appeared centrally hypofluorescent with a marginal irregular hyperfluorescence in the early arteriovenous phase and totally hyperfluorescent in the late venous phase. Interestingly, late-phase indocyanine green angiography disclosed a diffuse hypofluorescence in the macular region of both eyes. Optical coherence tomography (OCT) was abnormal only in the right eye, showing an anterior displacement of the photoreceptor layer by a hyperreflective structure located over a hyporeflective space above the retinal pigment epithelium layer.

No evidence of a familial inheritance pattern, an unusual OCT presentation and, above all, AOFVD onset shortly after the disclosure of acquired PAP prompted us to consider a not purely coincidental association between the two diseases.
Although no data about ocular tissues are available, animal models of PAP manifest a number of more subtle but important extrapulmonary abnormalities [2]. Furthermore, another study [3] has emphasised the role of GM-CSF, which is released by retinal pigment epithelial cells, as a regulator of macrophage and granulocyte function in the eye, and lipofuscin (the likely aetiology of the vitelliform appearance of the fovea in AOFVD) is thought to be the indigestible residues of rod outer segments that have been phagocytosed by macrophages [4]. Lastly, transgenic mice with dysregulated GM-CSF production exhibit a wide range of ocular lesions [5].

Ophthalmological investigations in other patients with pulmonary alveolar proteinosis, but not necessarily with ocular symptoms, could elucidate the possible relationship between this disease and macular disorders.

\section{P. Perri, C. Campa, S. D'Angelo, C. Costagliola, C. Incorvaia} and A. Sebastiani

Dept of Ophthalmology, University of Ferrara, Ferrara, Italy.

\section{REFERENCES}

1 Trapnell BC, Whitsett JA, Nakata K. Pulmonary alveolar proteinosis. N Engl J Med 2003; 349: 2527-2539.

2 Seymour JF, Presneill JJ. Pulmonary alveolar proteinosis: progress in the first 44 years. Am J Respir Crit Care Med 2002; 166: 215-235.

3 Crane IJ, Kuppner MC, McKillop-Smith S, Wallace CA, Forrester JV. Cytokine regulation of granulocytemacrophage colony-stimulating factor (GM-CSF) production by human retinal pigment epithelial cells. Clin Exp Immunol 1999; 115: 288-293.

4 Kennedy CJ, Rakoczy PE, Constable IJ. Lipofuscin of the retinal pigment epithelium: a review. Eye 1995; 9: 763-771.

5 Lang RA, Metcalf D, Cuthbertson RA, et al. Transgenic mice expressing a hemopoietic growth factor gene (GM-CSF) develop accumulations of macrophages, blindness, and a fatal syndrome of tissue damage. Cell 1987; 51: 675-686.

DOI: $10.1183 / 09031936.06 .00038906$ 